\title{
COMPARATIVE ANALYSIS OF VM PLACEMENT AND MIGRATION ALGORITHMS IN VM CONSOLIDATION
}

\author{
Rashmi Sindhu ${ }^{1}$, Vikas Siwach ${ }^{2}$, Harkesh Sehrawat ${ }^{{ }^{*}}$ \\ ${ }^{1}$ Research Scholar, ${ }^{2,3 *}$ UIET, Maharshi Dayanand University, Rohta \\ Email: sehrawat_harkesh@yahoo.com,
}

\begin{abstract}
With the increasing number of Internet of Things (IoT) devices, data centers are experiencing immense augmentation in the hardware devices with an increase in the traffic to the cloud infrastructures. To handle this growth and to satisfy users demand, data centers require more energy. The IoT devices produce vast data which needs to be handled properly by the data centers which in turn is responsible for increase in the power consumption at the data centers Management and reduction of this energy is quite a challenging task for the managers and the designers of the data centers as increasing cost of data centers is posing a major hindrance.. One major aspect that needs to be taken into consideration is the sharing of the data center resources which is fundamentally achieved by the consolidation of the resources. The analysis done will conclude that consolidation plays an important role in the reduction of energy consumption of a data center.
\end{abstract}

\section{Introduction}

With the rapid advancement in the technology, the IOT technology is also growing at an immense pace, as it offers marvellous opportunities across several fields such as public transport, personal vehicles and smart houses etc. The argument behind this is that the IOT prototype has improved our lives by providing smart solutions with prediction oriented capabilities [6]. Cloud computing is one among the several tools that guarantees the proper functioning of the IOT devices by ensuring their high performances, storage framework and processing of the IOT data in real time. Before the involvement of cloud in the computing, the computing services were managed by centralized servers. With the development and requirement, then comes the era of distributed computing where the consumer can access unlimited services at any place and at any time. Some IOT devices such as mobile devices continuously access the internet which produces large amount of data at the end of the network [7], which in turn increases the burden on the data centers. The applications that require response and processing in real time encounter the problem of more transmission delay due to the remote distance between the cloud data centre and the mobile devices. This problem is interpreted by the commencement of the edge computing which is introduced between the data center and the end devices. The edge computing can be characterized as the advancement over the cloud computing inspite of being alternator to the cloud computing. With the maturity of cloud computing technology, requirement of large data centers is also initiated. In the past decade, data centers had become the conventional source of providing IT services and internet services. The cloud computing service providers such as Amazon, Facebook, Microsoft, Google etc. are organising a number of data centers worldwide. In general a data center is referred as a single entity, but in actuality it is the combinations of three independent categories i.e. computation, storage and networking. These categories include a number of computational devices and servers which consume a staggering amount of power and also raise the operational cost of data centers.

According to a report generated by Research and Markets (World's largest market research store) on December 6, 2019 data centers markets are expected to grow up from $\$ 1,062.3$ million in 2018 to $\$ 1,950.0$ million by 2025 .

In this paper focus is on the power consumption parameter of the data centers and analysis of various techniques applied in different modules to reduce the overall power consumption of the data centers. Power consumption in a data center primarily depends on the consumption by the IT equipments such as servers and the cooling system. Figure 1 illustrates the distribution of the energy consumed by a data center. 

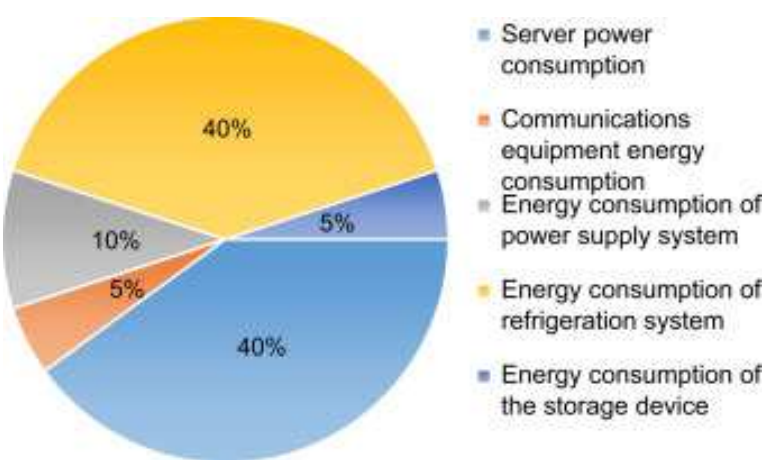

Figure 1: energy consumption by a data center

According to the Research and Markets report power and cooling segment of Indian data center this market is estimated to be $\$ 623.3$ million in 2019 and is expected to reach $\$ 1,0655.5$ million by 2025 . Researchers have suggested various ways of energy efficient consumption in a data center such as application scheduling, workload scheduling, resource scheduling, reducing the number of active networking switches, task scheduling etc.

A data center comprises a large number of physical machines (PMs) which are either arranged in various clusters or individual that can be homogenous or heterogeneous on the basis of resource configuration of the coming workload to the data centers which varies with time and with the type of application. A virtualized environment is embedded along with the PMs in the data center. Virtualization includes virtual resources such as CPU, memory storage that are configured with the operating system of the PMs. These virtual resources are termed as virtual machines (VMs) which are a type of software and are an emulation of a computer system based on computer architecture to provide functionality of a physical computer. The functioning and execution of VMs is same as of PMs. Every cloud service provider provides computing resources and services in the form of VM on the basis of Service Level Agreement (SLA). The main advantage of virtualization is that the client can change the resource utilisation according to their need as well as service providers can change their clients based on their usage in a payas-you-go-scheme [30]. Virtualization of resources in one or more data centers forms an efficient and a flexible resource pool that helps in reducing the infrastructure cost, reduction in the time to expend data centers also provide reliability to deal with changing business needs [5].

\section{VM Consolidation}

Multiple VMs can be embedded on a single PMs to achieve better resource utilisation and also to reduce the power consumption of data center by the consolidation of the VMs. The idea behind the consolidation of the VMs is to reduce the active PMs in the data center which can be achieved either statically or dynamically. In static VM consolidation, prior to the arrival of a workload the placement of
VMs over PMs is decided and cannot be changed over time wherelse in dynamic VM consolidation, a VM can be reallocated or migrated among several PM based on their requirement. This leads to the better utilization of the resources and also in reducing the power consumption of the data center.

The dynamic consolidation process is classified under into 4 major subcategories:-

1. State of resources: This fundamentally aims towards the availability and requirement of physical as well as virtual resources by an assigned workload.

2. Selection of VM: Based on the configuration of $\mathrm{PM}$, a VM is elected among various assigned VM to consolidate.

3. Placement of VM: After choosing a VM, it needs to be placed on a PM called host. Placement of a VM depends on the physical resources as CPU and storage. It can be either automatic or manual.

4. Migration of VM: VM migration is initiated when the VM placement shows inefficiency in reducing the power consumption or unavailability of resources or increase of overhead over the PM. Hence forth, placement of a VM is an initial part of VM migration.

This analysis includes the commonly used placement and migration algorithms which converge towards the efficiency of the energy.

Rest of the paper will briefly discuss the subcategories of dynamic VM consolidation and focus towards the few of placement and migration algorithms as well as characterization based on some widely used parameters of VM consolidation.

\section{Dynamic VM consolidation \\ State of resource scheme}

Data center can be abbreviated as a compound of heterogeneous PMs which possess heterogeneity in their resource requirement and inbuilt configuration. Thus power consumption of a PM is not constant and depends on its specification as well as on the load carried out by it. Therefore the state of physical resources before and after initial assignment of the VMs plays an important role in dynamic VM consolidation. Resource allocation is done by the scheduler to allocate the resources to fulfill a task from the queue of tasks. Scheduler on a system operates all tasks and resources and on the basis of the requirement, the scheduler decides either to provision a new VM to a PM or to allocate resources to an existing VM.

\section{Selection of $V M$}

It is a process of electing a VM from the pool of VMs that have been allocated to the servers, which need to be reallocated to the other servers in the data center. The purpose of reallocation is either to minimize the number of VMs or to minimize the number of PMs by switching the power off of inactive or unused PMs. Selection procedure comes into existence when 
the PM become overload or underload, in both the cases, the power consumption is reduced.

\section{VM placement}

Selection of VM is followed by its placement over a $\mathrm{PM}$. This mapping between the VM and PM is many to one, where every VM will have only one host PM, but a PM can have multiple VM embedded over it. This mapping is done to maximize the utilization of the resources and to efficiently use the power consumed by the data center by shutting down the unused PMs. When the mapping between the VM and the PM is fixed during the lifetime of the VM then it is known as static VM placement. While the initial placement of the VM can be changed according to the requirement or to revert from the undesired state of power, load and system performance is called dynamic VM placement.

Various researchers have suggested various algorithms either based on some problem in VM placement or on the optimization of the VM placement algorithms. About hundreds of VM placement algorithms have been suggested and each of the algorithm focuses on a different issue of placement. In this paper, focus is on few of the algorithms and their tabularization according to the proposed algorithm along with merit and demerit of the suggested algorithms.

T.Renugadevi et al.[10] had formulated the objective function with energy cost of both servers and cooling devices. Renewable-aware algorithms were evaluated with different parameters to determine the carbon intensity. Trfan Mohiuddin et al. [6] proposed WAVMCM ( Workload Aware VM Consolidation Method) to reduce the number of active PMs by consolidating VM to less number of PM. Zolten Mann et al. [18] experimentally compared the performance of seven different VM placement algorithms by contemplating parameters such as energy efficiency, characteristic of PMs, load prediction etc. Kansal et al.[19] emphasized on the resource allocation which affect much the need of consolidation although QoS (Quality of standards) gets satisfied. Wang et al. [17] suggested an Auction Based policy for VM allocation to the PM. Wangel et al. [20] highlighted the issue of unpredictable workload and proposed a ECRASP (Energy Conserving Resource Allocation Scheme with Prediction) for the allocation of VM. Vasu et al. [14] discussed load balancing and proposed the use of reliability record at server end but did not mention the implication of maintaining such record. Ali et al. [11] highlighted an energy efficiency algorithm and dealed with large data centers but this approach was not applicable on data centers with different sizes. Yanggratoke et al. [12] proposed a heuristic solution by taking load pattern into account for VM consolidation, but this solution was not effective in all sort of environment. Jha et al. [13] proposed a power and load aware VM allocation policy which was implemented and concluded without computational time required by the strategy. Table 1 lists some VM placement algorithms or approach with their merits and demerits that had been proposed by the earlier researchers.

\begin{tabular}{|c|c|c|c|}
\hline $\begin{array}{l}\text { Refer- } \\
\text { ence }\end{array}$ & Algorithm/ Approach & Improvement criteria & Demerit \\
\hline$[14]$ & FUSD (Fast Up Slow Down) algorithm & Maximum utilization & $\begin{array}{l}\text { CPU, storage and virtualized environment was } \\
\text { not considered }\end{array}$ \\
\hline$[16]$ & $\begin{array}{l}\begin{array}{l}\text { MBFD (Modified } \\
\text { algorithm }\end{array} \\
\end{array}$ & Energy Efficiency & \\
\hline$[17]$ & Auction strategy of VM allocation & Energy Efficiency & $\begin{array}{l}\text { No improvement was shown in dynamic } \\
\text { environment over other algorithms }\end{array}$ \\
\hline$[18]$ & DSAP (Dynamic Server Allocation Problem) & $\begin{array}{l}\text { Energy Efficiency and reduction in server } \\
\text { damage }\end{array}$ & Estimation of migration overhead is not correct \\
\hline [19] & Artificial Bee Colony Algorithm & Energy Efficiency and less execution time & Workload of nodes is not considered \\
\hline [20] & $\begin{array}{lrl}\text { ECRASP (Energy Conservation } & \text { Resource } \\
\text { Allocation Scheme with Prediction) } & \\
\end{array}$ & Improvement in Performance & $\begin{array}{l}\text { Only theoretical concept was proposed but not } \\
\text { implemented }\end{array}$ \\
\hline [11] & EE (Energy Efficient algorithm) & Performance Improvement & $\begin{array}{l}\text { Given algorithm need to be compared with } \\
\text { existing algorithms }\end{array}$ \\
\hline [12] & $\begin{array}{l}\text { GRMP (Generic Gossip protocol for Resource } \\
\text { Management) }\end{array}$ & Energy Efficiency & No comparison with other existing algorithms \\
\hline [13] & Power and Load aware VM allocation policy & Improvement in performance & Load Balancing was not considered \\
\hline [22] & $\begin{array}{l}\text { MWFDVP (Modified Worst Fit Decreasing VM } \\
\text { placement) algorithm }\end{array}$ & Energy Efficiency & $\begin{array}{l}\text { Single algorithm was considered for comparing } \\
\text { with other algorithms }\end{array}$ \\
\hline [22] & Practical Swarm Optimization algorithm & Energy Efficiency & No comparison \\
\hline [23] & A Gossip Protocol & Energy Efficiency & $\begin{array}{l}\text { Resource Allocation system is bounded to } \\
100000 \text { machines }\end{array}$ \\
\hline [24] & $\begin{array}{l}\text { Energy Aware Resource Provisioning Frame } \\
\text { algorithm }\end{array}$ & Energy Efficiency & Only cloud providers were taken into account \\
\hline [33] & $\begin{array}{l}\text { Distributed and Time-Hierarchical resource } \\
\text { management }\end{array}$ & $\begin{array}{l}\text { Minimizes SLA violation and reduces } \\
\text { energy consumption }\end{array}$ & Storage and memory was not considered \\
\hline [39] & Green Load Balancing policy & Load Balancing and Energy Efficiency & No concept of Virtualized environment was used \\
\hline
\end{tabular}

Table 1: Various VM placement algorithms 


\section{VM Migration}

VM migration is the process of shifting VM from one PM to another PM either to increase the resource capability of the available resources or to minimize the active number of PMs so that the overall power consumption of the data center can be reduced. Recent technologies accomplish the conception of seamless or live migration of VMs as they allow exclusive short downtime and migration time upto tens of millisecond [25]. Down time is the time taken to respond to a service and migration time is the time taken by the VM to migrate from a PM to the other PM.

Xiaodong $\mathrm{Wu}$ [29] et al. presented a scheduling algorithm based on combination of VM selection

policy and strategy of utilization of threshold as well as experimentally evaluated that number of VM migration were reduced, thus lower energy consumption by the data center. Hiroki Shiranyangi et al. [27] highlighted an energy efficient network topology named Honeyguide which combines VM consolidation with bypass links. Using simulator tool, it was proved that this strategy result in less power consumption. Srinivas Byatarayanapura Venkataswamy et al. [26] proposed an optimized VM migration algorithm named ChicWhale by considering factors like load, resource availability, and energy consumed and migration cost.
The proposed algorithm was advised to use at medical Image Quality Enhancement System etc. Li et al. [ 31] explained Dynamic VM consolidation based on multi-resource energy efficient model. It emphasized on distributing the load among the computing servers to fulfil the user requirement. Geo et al. [34] proposed a multiobjective Ant Colony System algorithm by keeping power consumption and resource utilization under consideration but showed poor results when the data sets were large. Yermolovich et al. [38] discussed an optimizing trace - based VM policy which depends on the execution paths of the VMs that were stored and used for other VMs for migration but results in magnification of processing time. Patel et al. [36] proposed a Dolphin Echolocation technique which reduces the power consumption and

wastage of resources but increases the migration overhead. Narantuya et al. [37] presented ServiceAware strategy for $\mathrm{C} 2 \mathrm{C}$ migration, that focused on reducing the service downtime. This strategy did not mention about the destination where the VM is being migrated. Paulraj et al. [35] proposed a resourceaware VM migration technique and experimentally concluded that this technique reduces the downtime, end to end delay and migration time. Table 2 list some VM migration algorithms or approaches along with their merits and their demerits.

\begin{tabular}{|l|l|l|l|}
\hline $\begin{array}{l}\text { Refere- } \\
\text { nce }\end{array}$ & Algorithm/Approach & Improvement criteria & Demerit \\
\hline$[31]$ & $\begin{array}{l}\text { Dynamic energy efficient VM } \\
\text { migration and consolidation algorithm }\end{array}$ & Energy Efficiency & Increases the service downtime \\
\hline$[32]$ & 2-tier VM placement algorithm & $\begin{array}{l}\text { Resource utilisation and energy } \\
\text { efficiency }\end{array}$ & $\begin{array}{l}\text { More storage requirement as VM } \\
\text { size increases }\end{array}$ \\
\hline$[34]$ & Ant Colony System algorithm & $\begin{array}{l}\text { Resource utilisation and energy } \\
\text { efficiency }\end{array}$ & Fails on large data sets \\
\hline$[35]$ & $\begin{array}{l}\text { Resource-Aware VM migration } \\
\text { technique }\end{array}$ & $\begin{array}{l}\text { Less migration time, downtime } \\
\text { and end to end delay }\end{array}$ & Algorithm is not optimized \\
\hline$[37]$ & $\begin{array}{l}\text { Service- Aware strategy for C2C } \\
\text { migration }\end{array}$ & Less service downtime & $\begin{array}{l}\text { Destination host of VM is not } \\
\text { mentioned }\end{array}$ \\
\hline$[38]$ & Optimizing trace based VM & $\begin{array}{l}\text { Stores the execution path of } \\
\text { VM }\end{array}$ & Requires more processing time \\
\hline$[36]$ & Dolphine Echolocation Technique & $\begin{array}{l}\text { Energy Efficiency and } \\
\text { Resource utilization }\end{array}$ & Migration cost overhead \\
\hline
\end{tabular}

Table 2: Various VM migration algorithms

\section{Conclusion and Future Scope}

Based on the analysis don so far, it is not appropriate to justify the best algorithm for VM placement and VM migration as each algorithm has its own merit and demerit. Every algorithm uses a different criterion for placement and migration of the VMs and those algorithms which uses the same criteria shows improvement in distinct parameters of the data center. In future this analysis will be followed either by combining the two techniques for VM placement and migration or by introducing a new technique that aim towards the reduction of power consumption of the data center. Also while placing and migration of VM, configuration of PM will also be considered as 
it plays a vital role during the entire consolidation process.

\section{References}

1. V. S. Shekhawat, A. Gautam and A. Thakrar, "Datacenter Workload Classification and Characterization: An Empirical Approach," 2018 IEEE 13th International Conference on Industrial and Information Systems (ICIIS), Rupnagar, India, 2018, pp. 1-7, doi: 10.1109/ICIINFS.2018.8721402.

2. Lin, Ruihong \& Deng, Yuhui. (2016). Allocating workload to minimize the power consumption of data centers. Frontiers of Computer Science. 11. 10.1007/s11704016-6035-z.

3. T. Adhikary, A. K. Das, M. A. Razzaque and A. M. J. Sarkar, "Energy-Efficient Scheduling Algorithms for Data Center Resources in Cloud Computing," 2013 IEEE 10th International Conference on High Performance Computing and Communications \& 2013 IEEE International Conference on Embedded and Ubiquitous Computing, Zhangjiajie, China, 2013, pp. 1715-1720, doi: 10.1109/HPCC.and.EUC.2013.244.

4. M. I. K. Khalil, I. Ahmad and A. A. Almazroi, "Energy Efficient Indivisible Workload Distribution in Geographically Distributed Data Centers," in IEEE Access, vol. 7, pp. 82672-82680, 2019, doi: 10.1109/ACCESS.2019.2924085.

5. Zhang, Weizhe \& He, Hui \& Chen, Gui \& Sun, Jilong. (2013). Multiple Virtual Machines Resource Scheduling for Cloud Computing. Applied Mathematics \& Information Sciences. 7. 2089-2096. 10.12785/amis/070551.

6. Bangui, Hind \& Rakrak, Said \& Raghay, Said \& Buhnova, Barbora. (2018). Moving to the Edge-Cloud-of-Things: Recent Advances and Future Research Directions. 7. 309. 10.3390/electronics7110309.

7. Li, Guangshun \& Song, Jianrong \& Wu, Junhua \& Wang, Jiping. (2018). Method of Resource Estimation Based on QoS in Edge Computing. Wireless Communications and Mobile Computing. 2018. 1-9. 10.1155/2018/7308913.

8. Mann, Zoltan \& Szabó, Máté. (2017). Which is the best algorithm for virtual machine placement optimization? Concurrency and Computation: Practice and Experience. 29. e4083. 10.1002/cpe.4083.

9. Mohiuddin, Irfan \& Almogren, Ahmad. (2018). Workload aware VM consolidation method in edge/cloud computing for IoT applications. Journal of Parallel and
Distributed Computing.

123. 10.1016/j.jpdc.2018.09.011.

10. Renugadevi, T.; Geetha, K.; Muthukumar, K.; Geem, Zong W. 2020. "Optimized Energy Cost and Carbon Emission-Aware Virtual Machine Allocation in Sustainable Data Centers" Sustainability 12, no. 16: 6383. https://doi.org/10.3390/su12166383

11. A. Ali, L. Lu, Y. Zhu and J. Yu, "An Energy Efficient Algorithm for Virtual Machine Allocation in Cloud Datacenters," in ACA 2016: Advanced Computer Architecture. Communications in Computer and Information Science, Singapore, 2016.

12. R. Yanggratoke, F. Wuhib and R. Stadler, "Gossip-based resource allocation for green computing in large clouds," in Proceedings of the $7^{\text {th }}$

International Conference on Network and Services Management, Paris, France, 2011

13. R. S. Jha and P. Gupta, "Power aware resource allocation policy for hybrid cloud," 2015 Third International Conference on Image Information Processing (ICIIP), pp. 1-45, 2015.

14. R. Vasu, E. I. Nehru and G. Ramakrishnan, "Load Forecasting for Optimal Resource Allocation in Cloud Computing Using Neural Method," Middle-East Journal of Scientific Research, vol. 24, no. 6, pp. 19952002, 2016.

15. A.-p. Xiong and C.-X. Xu, "Energy Efficient Multiresource Allocation of Virtual Machine Based on PSO in Cloud Data Center," Mathemtatical Problems in Engineering, vol. 2014, pp. 1-8, 2014.

16. Anton Beloglazov and Rajkumar Buyya. Optimal online deterministic algorithms and adaptive heuristics for energy and performance efficient dynamic consolidation of virtual machines in cloud data centers. Concurrency and Computation: Practice and Experience, 24(13):1397-1420, 2012.

17. W. Wang, Y. Jiang and W. Wu, "Multiagent-Based Resource Allocation for Energy Minimization in Cloud Computing Systems," IEEE Transactions on Systems, Man, and Cybernetics: Systems, vol. 47, no. 2, pp. 205-220, 2016.

18. A. Wolke and L. Ziegler, "Evaluating Dynamic Resource Allocation Strategies in Virtualized Data Centers," in 2014 IEEE 7th International Conference on Cloud Computing (CLOUD), 2014.

19. N. J. Kansal and I. Chana, "Artificial bee colony based energyaware resource utilization technique for cloud computing," Journal of Concurrency and Computation: 
Practice \& Experience, vol. 27, no. 5, pp. 1207-1225, 2015.

20. C.-F. Wang, W.-Y. Hung and C.-S. Yang, "A Prediction Based Energy Conserving Resources Allocation Scheme for Cloud Computing," in IEEE International Conference on Granular Computing (GrC), 2014.

21. H. Hlavacs and Treutner, "Genetic algorithms for energy efficient virtualized data centers," in Proceedings of the 2012 8th International Conference on Network and Service Management (CNSM) and 2012 Workshop on Systems Virtualiztion Management (SVM), pp. 422-429, Las Vegas, NV, USA,October 2012.

22. Mohammed Rashid Chowdhury, Mohammad Raihan Mahmud, and Rashedur M. Rahman. Study and performance analysis of various VM placement strategies. In 16th IEEE/ACIS International Conference on Software Engineering, Artificial Intelligence, Networking and Parallel/Distributed Computing, page DOI: 10.1109/SNPD.2015.7176234, 2015.

23. F. Wuhib, R. Yanggratoke and R. Stadler, "Allocating Compute and Network Resources Under Management Objectives in Large-Scale Clouds," Journal of Network and Systems Management, vol. 23, no. 1, pp. 111-136, 2015.

24. M. Dabbagh, B. Hamdaoui and M. Guizani, "Energy-Efficient Resource Allocation and Provisioning Framework for Cloud Data Centers," IEEE Transactions on Network and Service Management, vol. 12, no. 3, pp. 377-391, 2015.

25. Wang, Xiaoying \& Liu, Xiaojing \& Fan, Lihua \& Jia, Xuhan. (2013). “A Decentralized Virtual Machine Migration Approach of Data Centers for Cloud Computing". Mathematical Problems in Engineering. 2013. 10.1155/2013/878542.

26. Venkataswamy, Srinivas \& Mandal, Indrajit \& Keshavarao, Seetharam. (2020). "ChicWhale optimization algorithm for the VM migration in cloud computing platform". Evolutionary Intelligence. 13. 10.1007/s12065-020-00386-9.

27. Shirayanagi, Hiroki \& Yamada, Hiroshi \& Kono, Kenji. (2012).’Honeyguide: A VM Migration-Aware Network Topology for Saving Energy Consumption in Data Center Networks". Proceedings - International Symposium on Computers and Communications. E96D. 000460-000467. 10.1109/ISCC.2012.6249339.

28. Beloglazov, Anton \& Buyya, Rajkumar. (2014). OpenStack Neat: A framework for dynamic and energy-efficient consolidation of virtual machines in OpenStack clouds. Concurrency and Computation: Practice and Experience. 27. 10.1002/cpe.3314.

29. Alshayeji, Mohammad \& Abed, Sawsan \& Samrajesh, M.. (2017). "Energy Efficient Virtual Machine Migration algorithm". Journal of Engineering Research. 5. 19-42.

30. Ismaeel, Salam \& Karim, Raed \& Miri, Ali. (2018). "Proactive dynamic virtual-machine consolidation for energy conservation in cloud data centres". Journal of Cloud Computing. 7. 10.1186/s13677-018-0111-x.

31. Huang W, Ma Z, Dai X, Mingdi Xu, Gao Yi (2018) "Fuzzy clustering with feature weight preferences for load balancing in cloud". Int J Softw Eng Knowl Eng 28(5):593-617.

32. Jansen W, Grance T (2011) "Guidelines on security and privacy in public cloud computing", pp 800-144.

33. M. Guazzone, C. Anglano, and $M$. Canonico. "Exploiting VM migration for the automated power and performance management of green cloud computing systems". In 1st International Workshop on Energy Efficient Data Centers, pages 81-92, 2012.

34. Gao Y, Guan H, Qi Z, Hou Y, Liu L (2013) "A multi-objective ant colony system algorithm for virtual machine placement in cloud computing". J Comput Syst Sci 79(8):1230-1242.

35. Milani AS, Navimipour NJ (2016) "Load balancing mechanisms and techniques in the cloud environments: Systematic literature review and future trends". J Netw Comput Appl 71(1):86-98.

36. Patel RP (2019) "Energy efficient VM migration in cloud datacenter using Dolphin echolocation optimization with Tchebycheff algorithm". Int J Recent Technol Eng (IJRTE) 8(2):86-94.

37. Li H, Zhu G, Cui C, Tang H, Dou Y, He C (2016) "Energy-efficient migration and consolidation algorithm of virtual machines in data centers for cloud computing". Computing 98(3):303-317.

38. Yermolovich A, Wimmer C, Franz M (2009) "Optimization of dynamic languages using hierarchical layering of virtual machines". In: Proceedings of the 5th symposium on Dynamic languages, pp 7988.

39. Hongjian Li, Guofeng Zhu, Chengyuan Cui, Hong Tang, Yusheng Dou, and Chen He. "Energye efficient migration and consolidation algorithm of virtual machines in data centers for cloud computing". Computing, 98(3):303-317, 2016. 